\title{
Metallomics
}

Cite this: Metallomics, 2014 6,1079

Received 7th January 2014 Accepted 26th March 2014

DOI: $10.1039 / \mathrm{c} 4 \mathrm{mt} 00004 \mathrm{~h}$

www.rsc.org/metallomics

\section{Hypoxia reduces and redirects selenoprotein biosynthesis $\dagger$}

\author{
Niels-Peter Becker, ${ }^{a}$ Janine Martitz, ${ }^{a}$ Kostja Renko, ${ }^{a}$ Mette Stoedter, ${ }^{a}$ \\ Sandra Hybsier, ${ }^{a}$ Thorsten Cramer $^{b}$ and Lutz Schomburg*a
}

\begin{abstract}
Selenium deficiency constitutes a risk factor for the incidence and negative course of severe diseases including sepsis, stroke, autoimmune diseases or cancer. In this study, hypoxia is identified as a powerful stimulus to redirect selenoprotein biosynthesis causing reduced selenoprotein $P$ expression and diminished selenium export from hepatocytes in favour of increased biosynthesis of the essential protective intracellular phospholipid hydroperoxide glutathione peroxidase GPX4. Specifically, hypoxia decreases transcript concentrations of central factors controlling selenium and selenocysteine metabolism including selenophosphate synthetase-2, phosphoseryl-tRNA ${ }^{\text {SerSec }}$ kinase and selenocysteine lyase, which are all proven to be rate-limiting enzymes in selenoprotein biosynthesis. These effects are paralleled by a general decline of selenoprotein expression; however, not all selenoproteins are affected to the same extent by hypoxia, and GPX4 constitutes an exception as its expression becomes slightly increased. Supplemental selenium is able to overcome the hypoxia-dependent down regulation of selenoprotein expression in our cell culture model system, supporting the concept of using selenium as an adjuvant treatment option in severe diseases. Although it remains to be tested whether these effects constitute a hepatocyte-specific response, the selenium-dependent decline of selenoprotein $P$ biosynthesis under hypoxic conditions may explain the progressive selenium deficit developing in severe diseases.
\end{abstract}

\section{Introduction}

Selenium (Se) is an essential trace element needed for the biosynthesis of a limited number of selenoproteins controlling central metabolic pathways in eukaryotes. ${ }^{1}$ Biosynthesis of selenoproteins is hierarchically regulated ensuring that the most important Se-dependent processes are maintained even under conditions of severe Se deficiency. ${ }^{2}$ Selenoproteins fulfil important functions in thyroid hormone homeostasis, protection from oxidative stress, immune response, redox-related signalling and quality control of secreted or aged proteins. ${ }^{3}$ Accordingly, selenium (Se) deficiency increases the risk of a number of common human pathologies, e.g., cancer, ${ }^{4}$ infections,${ }^{5}$ cognitive decline,${ }^{6}$ cerebrovascular events ${ }^{7}$ or thyroid diseases. ${ }^{8}$ Importantly, some experimental studies and clinical trials have reported positive effects of Se supplementation on the disease course and outcome in patients with severe sepsis, ${ }^{9}$ autoimmune thyroid disease ${ }^{8}$ stroke $^{10}$ or cardiac arrest. ${ }^{11}$ The plethora of interactions

\footnotetext{
${ }^{a}$ Institute for Experimental Endocrinology, Charité-Universitätsmedizin Berlin, CVK, D-13353 Berlin, Germany. E-mail: lutz.schomburg@charite.de; Fax: +4930 450524922; Tel: +4930450524289

${ }^{b}$ Medizinische Klinik mit Schwerpunkt Hepatologie und Gastroenterologie, Charité-Universitätsmedizin Berlin, CVK, D-13353 Berlin, Germany $\dagger$ Electronic supplementary information (ESI) available. See DOI: 10.1039/ c4mt00004h
}

between the Se status, selenoproteins and common diseases can only hardly be reconciled with the current knowledge given the diverse underlying disease mechanisms and molecular pathways involved. We reasoned that an insufficient perfusion of the affected tissues may represent a common underlying motif of this set of apparently unrelated pathologies. Hypoxia might constitute a common stimulus for adapting Se metabolism and selenoprotein expression to the actual needs of the cells under low oxygen supply. Our results support this assumption and highlight a strong negative effect of hypoxia on the expression of selenoproteins and central factors of the selenoprotein biosynthesis machinery. We have chosen hepatocytes to study this novel pathway in order to better understand why systemic Se concentrations decline in severe diseases, as hepatocytes constitute the central cell type translating dietary Se sources into circulating selenoprotein P (SePP) as a systemic Se transporter accounting for the major fraction of Se in blood.

\section{Material and methods}

Cell culture and treatments

Human hepatoma HepG2 cells were grown at $37{ }^{\circ} \mathrm{C}, 5 \% \mathrm{CO}_{2}$ in DMEM/Ham's F-12 supplemented with 10\% FBS (fetal bovine serum), $2.5 \mathrm{mM}$ L-glutamine, and 5\% penicillin and streptomycin 
(Invitrogen, Darmstadt, Germany). Cells were seeded and treated with sodium selenite $\left(\mathrm{Na}_{2} \mathrm{SeO}_{3}\right)$ or Geneticin (G418) in FBS and phenol red-free medium as indicated. Selenite supplementation experiments were conducted by adding the trace element to the cell cultures at room temperature and under ambient conditions. Then, the cultures were placed in the incubators under normoxic or hypoxic conditions for a given period of time. Media were not changed and cell cultures were not manipulated during the incubation times. Regulation of hypoxia-responsive genes was determined to verify hypoxia; pericellular oxygen levels have not been determined in these experiments. At the end of the incubation periods, cells were removed once, immediately frozen or homogenized, and changes in selenoprotein expression levels were analysed. In order to determine SePP expression, $10 \mu \mathrm{l}$ of cell culture supernatants were collected, heat denaturated, separated by SDS-PAGE and blotted. The effects of glutamine (Q) on SePP expression were tested by adding increasing concentrations $(0,0.2$ and $2.0 \mathrm{mM}$, f.c.) of the amino acid $\mathrm{Q}$ to the cell culture medium before incubation under normoxic or hypoxic conditions (Fig. S1, ESI $\dagger$ ).

\section{Quantitative reverse transcription-PCR (qRT-PCR)}

Total RNA extractions were performed using the TRIzol ${ }^{\mathbb{R}}$ Reagent (PEQLAB, Erlangen, Germany) according to the manufacturer's instructions. RNA was further purified by one round of chromatography using a QIAGEN RNeasy mini column (QIAGEN, Hilden, Germany). RNA samples (500 ng) were reverse-transcribed or not (controls) using the iScript ${ }^{\mathrm{TM}}$ Select cDNA Synthesis Kit (BIO-RAD, Munich, Germany). QRT-PCR analyses were performed using the iCycler-System (BIO-RAD) and ABsolute qPCR SYBR Green Fluorescein Mix (Thermo Scientific, Schwerte, Germany). Melting curves were recorded at the end of each qRT-PCR analysis verifying the specificity of primer pairs and amplicons. Results represent mean values of $n=4$ reactions, and were normalized to $18 \mathrm{~S}$ rRNA. Gene expression was considered to be absent when specific amplicons were not obtained after 40 cycles of qRT-PCR. Primer sequences for SePP, DIO1, GPX1,-2,-4, eEFSec, SBP2, SCLY, PSTK, SEPHS2, SEPSECS, PGK1, CA9, and 18S rRNA are listed (Table S1, ESI $\dagger$ ). Ct values of the qRT-PCR analyses are provided in the ESI $\dagger$ (Table S2).

\section{Enzyme assays and SePP ELISA}

The enzymatic activity of the cytosolic glutathione peroxidases (GPX1, GPX2) was measured using tert-Butyl-hydroperoxide ( $t$ Butyl-OOH) as described in ref. 12. Membrane associated GPX4 was assessed by using cumene hydroperoxide as the substrate according to Lawrence and Burk. ${ }^{13}$ Deiodinase activity was assayed as described in ref. 14. SePP concentrations were quantified by a SePP-specific ELISA (Selenotest ${ }^{\mathrm{TM}}$, ICI immunochemical intelligence $\mathrm{GmbH}$, Berlin, Germany), related to the luminometric assay described earlier. ${ }^{15}$

\section{Western blot analyses}

Cells were washed with PBS and harvested in homogenization buffer (0.25 M sucrose; 20 mM HEPES; 1 mM EDTA, pH 7.4).
Cytosolic fractions were obtained after centrifugation for $10 \mathrm{~min}$ at $14000 \mathrm{rpm}$ and $4{ }^{\circ} \mathrm{C}$. Pellets were resuspended and solubilized in RIPA buffer (50 mM Tris/HCl $\mathrm{pH} 8.0$; $150 \mathrm{mM} \mathrm{NaCl} ; 1 \%$ NP-40; 0.5\% Na-deoxycholate; $0.1 \%$ SDS) to obtain the membrane fractions. Protein concentrations were quantified using a BIO-RAD Protein Assay (BIO-RAD, Munich, Germany) or a Pierce ${ }^{\circledR}$ BCA Protein Assay Kit (Thermo Scientific, Schwerte, Germany) using BSA as standard. Equal volumes of medium were diluted with $4 \times$ sample buffer (200 mM Tris-HCL, pH7.5; 50\% glycerin; 4\% SDS; $0.04 \%$ bromphenol blue and $125 \mathrm{mM}$ DTT). Samples were size fractionated by SDS-PAGE and blotted by semi-dry transfer onto nitrocellulose membranes (Optitran, Schleicher \& Schuell, Dassel, Germany). Antibodies against SePP (1:2000 dilution, ICI Diagnostics, Berlin), ceruloplasmin (1:2000 dilution, Abcam), GPX1 (1:1000 dilution, Abcam), beta-actin (1:25000 dilution, Roche), GPX4 (1:1000 dilution, Abcam), and DIO1 (1:1000 dilution, kind gift of Dr Georg Kuiper, Rotterdamm) were used and quantified using ECL ${ }^{\mathrm{TM}}$ Western Blotting Detection Reagents (GE Healthcare, UK). Quantification of Western blot signals was assessed using ImageJ (NIH, USA).

\section{Reporter assays and plasmids}

Reporter gene constructs were transfected and assayed as described in ref. 16. Efficiency of Sec incorporation was determined by the pBLUGA system ${ }^{17}$ using GPX4 and SePP SECIS constructs as templates. For each experiment 20000 HepG2 cells were seeded into 96 well plates and transfected with $95 \mathrm{ng}$ of the reporter and $5 \mathrm{ng}$ of the pGL4.74 renilla plasmid per well using the Fugene HD reagent (Promega, Mannheim, Germany). After 24 h, cells were washed with PBS and treated with 0, 10 or $100 \mathrm{nM}$ Na-selenite for $24 \mathrm{~h}$. Lysis of cells was achieved using $20 \mu \mathrm{l}$ of Passive Lysis Buffer (Promega). Activity was determined using the Luciferase ${ }^{\circledR}$ Reporter System (Promega) in combination with a Mithras LB 940 luminometer (Berthold Technologies, Bad Wildbad, Germany). Readings of firefly luciferase (Fluc) were normalized by the signals obtained for secreted alkaline phosphatase (Seap) as described in ref. 16 in order to correct for transfection and harvesting efficiencies and general effects on the biosynthesis rate or detection efficiency. Plasmids carrying constitutively active hypoxia-inducible factors (HIF), HIF1 $\alpha$ (P402A/P564A) and HIF2 $\alpha$ (P405A/P531A), were a kind gift of William G. Kaelin18 and distributed by Addgene (Addgene plasmids \#18955 and \#18956). Active HIF variants were cloned into the pCDH vector (catalog \# CD510B-1, System Biosciences, William San Francisco, USA) using BamH1 and Not1 restriction sites. The $\triangle$ ARNT plasmid was a kind gift of Prof. M. Schwarz (University of Tübingen, Germany).

\section{Statistics}

Statistical analyses were performed using GraphPad Prism v.4.0 (GraphPad Software Inc., San Diego, USA). Results are represented as means \pm SD or SEM. The number of replicates is given in the respective figure legends. Two group comparisons 
were conducted using Student's $t$-test. Statistical significance was assigned if $P<0.05\left({ }^{*}\right), P<0.01\left({ }^{* *}\right)$ or $P<0.001\left({ }^{* *}\right)$.

\section{Results}

\section{Hypoxia down-regulates selenoprotein expression}

Initially, the effects of hypoxia on selenoprotein expression were tested by comparing mRNA and protein concentrations of SePP and deiodinases type 1 (DIO1) in HepG2 cells under normoxic $\left(20 \% \mathrm{O}_{2}\right)$ versus hypoxic $\left(1 \% \mathrm{O}_{2}\right)$ conditions using two different Se concentrations (Fig. 1). As expected, SePP concentrations in culture medium were increased by supplementation with $100 \mathrm{nM}$ Na-selenite $\left(\mathrm{Na}_{2} \mathrm{SeO}_{3}\right)$ or in the presence of geneticin (G418), which is an aminoglycoside antibiotic known to suppress UGA termination codons of selenoproteins. ${ }^{18}$ Notably, besides selenite and geneticin, the glutamine (Q) supply also turned out to constitute an important limiting factor for SePP biosynthesis under both hypoxic and normoxic conditions (Fig. S1, ESI $\dagger$ ). Under hypoxic conditions, SePP concentrations were marginal as compared to normoxia, and the stimulatory effects of $\mathrm{Na}_{2} \mathrm{SeO}_{3}$ or G418 on SePP expression were strongly diminished (Fig. 1A). Specificity of the effects was verified by analysing ceruloplasmin (CP) in parallel as an unrelated secreted hepatic trace element transporter (Fig. 1A).

No consistent effects of $\mathrm{Na}_{2} \mathrm{SeO}_{3}, \mathrm{G} 418$ or hypoxia on CP concentrations were noted. On the transcript level, $100 \mathrm{nM}$ selenite exerted a strong positive effect on SePP mRNA levels under normoxic but only a moderate effect under hypoxic conditions (Fig. 1C). The strongly diminished SePP protein levels in hypoxia were not reflected by respective changes in SePP mRNA concentrations indicating that the posttranscriptional regulation of SePP biosynthesis is affected. This notion was supported when DIO1 as a second important selenoprotein was analysed. DIO1 enzymatic activity was two times higher under normoxic as compared to hypoxic conditions (Fig. 1D). Selenite supplementation increased DIO1 protein levels efficiently in normoxia but not in hypoxia (Fig. 1E). In comparison, only small effects of altered oxygen or selenite supply were noted on the DIO1 transcript level, and the changes observed do not align with the altered DIO1 protein expression (Fig. 1F). These findings indicate that hypoxia impairs the biosynthesis of selenoproteins at the posttranscriptional level.

\section{Isozyme-specific effects of hypoxia on glutathione peroxidases}

Hypoxia increases hydrogen peroxide concentrations and leads to an enhanced generation of reactive oxygen species. ${ }^{19}$ Therefore, we tested the effects of hypoxia on the expression of GPX isozymes implicated in peroxide degradation. GPX activity was tested using two different substrates, i.e., water soluble $t$ Butyl$\mathrm{OOH}$ being efficiently degraded by many GPX isozymes (GPX1, GPX2 and 3), and cumene hydroperoxide as the lipid substrate preferentially degraded by GPX $4 .{ }^{13}$ GPX activity against $t$ Butyl$\mathrm{OOH}$ as the substrate increased upon selenite supplementation as expected. However, the increase was twice as high under normoxia as compared to hypoxia (Fig. 2A). GPX1 transcript
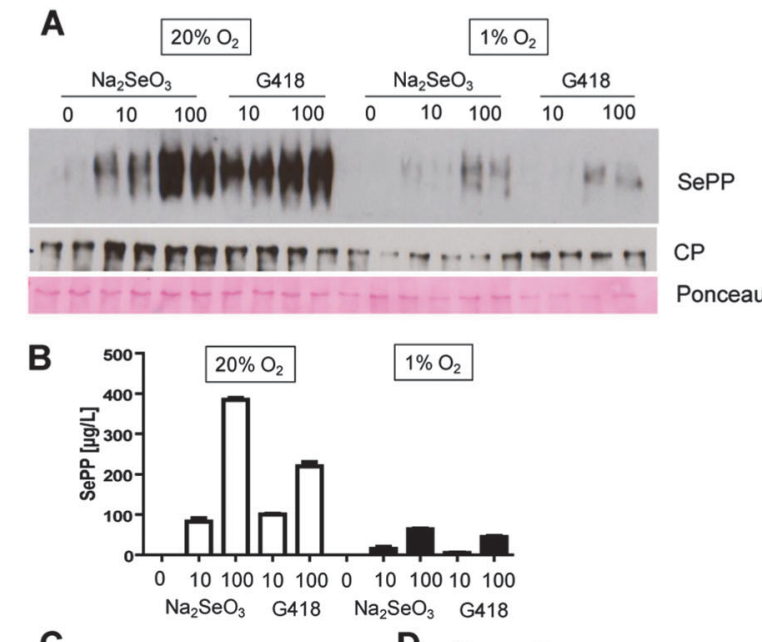

C
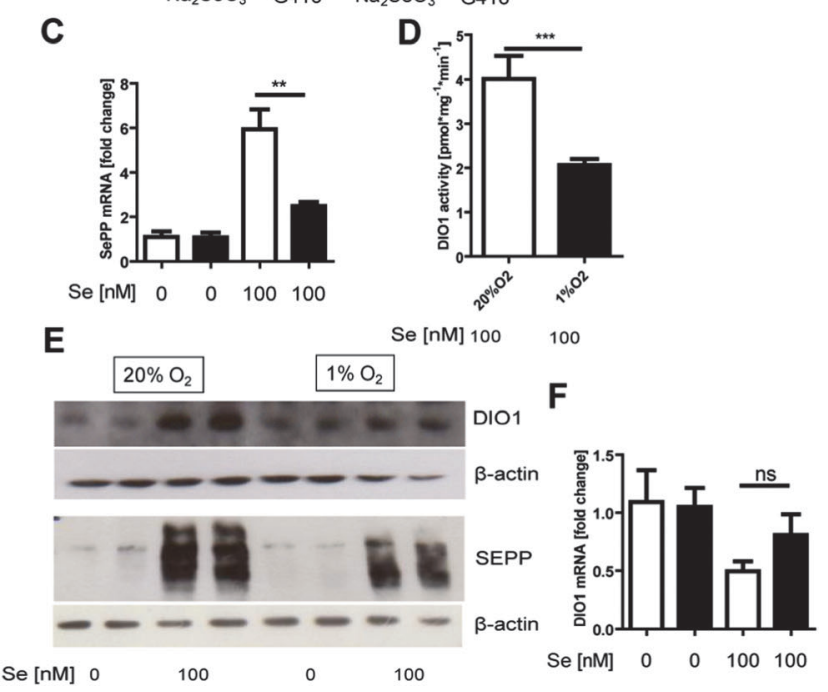

Fig. 1 Hypoxia affects selenoprotein $P$ (SePP) and deiodinases type 1 (DIO1) expression. (A) SePP concentrations increase in HepG2 medium after incubation with increasing amounts of $\mathrm{Na}$-selenite or geneticin (G418) under normoxic conditions. In hypoxia, the effects are mitigated. Effects on ceruloplasmin (Cp) expression are marginal under these conditions. (B) Quantification of SePP amounts from cell culture supernatants by ELISA are in agreement with the Western blot results. (C) SePP mRNA expression is induced by selenite under normoxic (open bars), but only moderately affected under hypoxic (black bars) conditions. (D) DIO1 enzymatic activity is lower in hypoxia as compared to normoxia in cultures containing $100 \mathrm{nM}$ selenite; without supplemental Se, DIO1 activity was below the detection limit. (E) Accordingly, intracellular DIO1 protein expression becomes strongly induced by selenite under normoxic, but not under hypoxic conditions. Similarly, intracellular SePP protein concentrations are strongly increased by selenite under normoxia but not under hypoxia. (F) Effects of hypoxia on DIO1 mRNA concentrations are relatively subtle and not in line with the differences in enzymatic activity. Representative data from $n=2-4$ similar experiments each involving at least duplicate determinations are shown.

levels increased upon supplementation with $100 \mathrm{nM}$ selenite irrespective of the oxygen status (Fig. 2B), whereas GPX1 protein concentrations declined under low oxygen (Fig. 2C). In comparison, hypoxia caused strongly increased GPX4 mRNA levels (Fig. 2D). In line with GPX4 mRNA, GPX activity measured with cumene hydroperoxide as the substrate and GPX4 protein levels increased under hypoxic conditions (Fig. 2E and F), 
A

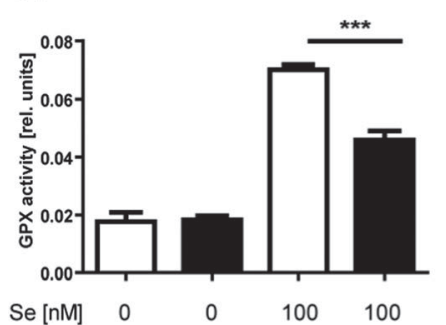

B

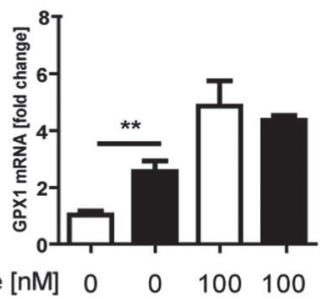

C

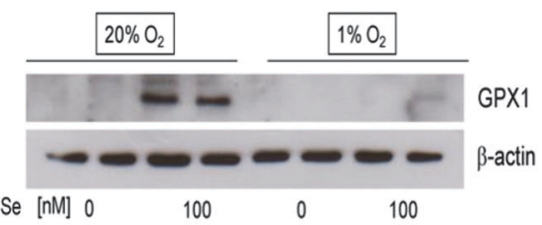

D

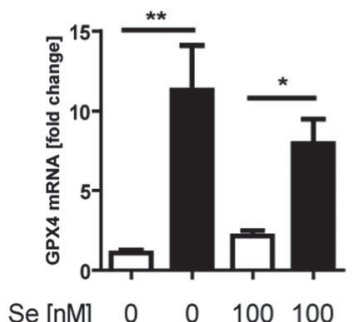

E

$\mathbf{F}$

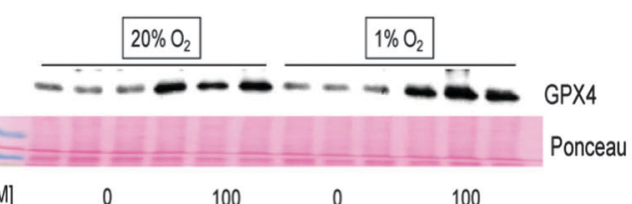

Se $[\mathrm{nM}]$

100

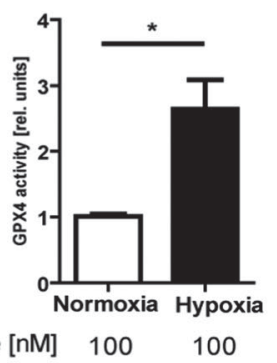

Fig. 2 Glutathione peroxidase (GPx) isozymes are differentially affected by hypoxia. (A) Selenite supplementation increases total GPX activity (measured with $t$ Butyl-OOH). The inducing effect is stronger under normoxia than under hypoxia. (B) GPX1 mRNA levels are higher under hypoxia than under normoxia, and increase upon selenite treatment further irrespective of oxygen availability. (C) GPX1 protein levels are strongly up regulated by selenite under normoxic, but not under hypoxic conditions (D) GPX4 mRNA concentrations, (E) GPX4 enzyme activity and (F) GPX4 protein levels are significantly up regulated by hypoxia in the presence of supplemental Se. Representative data from $n=2-4$ similar experiments each involving at least duplicate determinations are shown.

indicating GPX-isoform specific effects. However, the increase in GPX4 protein level was marginal in view of the several-fold higher GPX4 transcript concentrations under hypoxic conditions, verifying the notion that hypoxia reduced net selenoprotein biosynthesis by posttranscriptional mechanisms. There was no detectable GPX activity in the cell culture supernatants indicating the absence of GPX3 expression in these experiments.

\section{Role of SECIS-elements and HIF signalling in the hypoxic regulation of GPX4 and SePP}

As biosynthesis of selenoproteins critically depends on the SECIS elements and hypoxia mainly signals via HIF, we tested the relevance of these two factors for selenoprotein biosynthesis
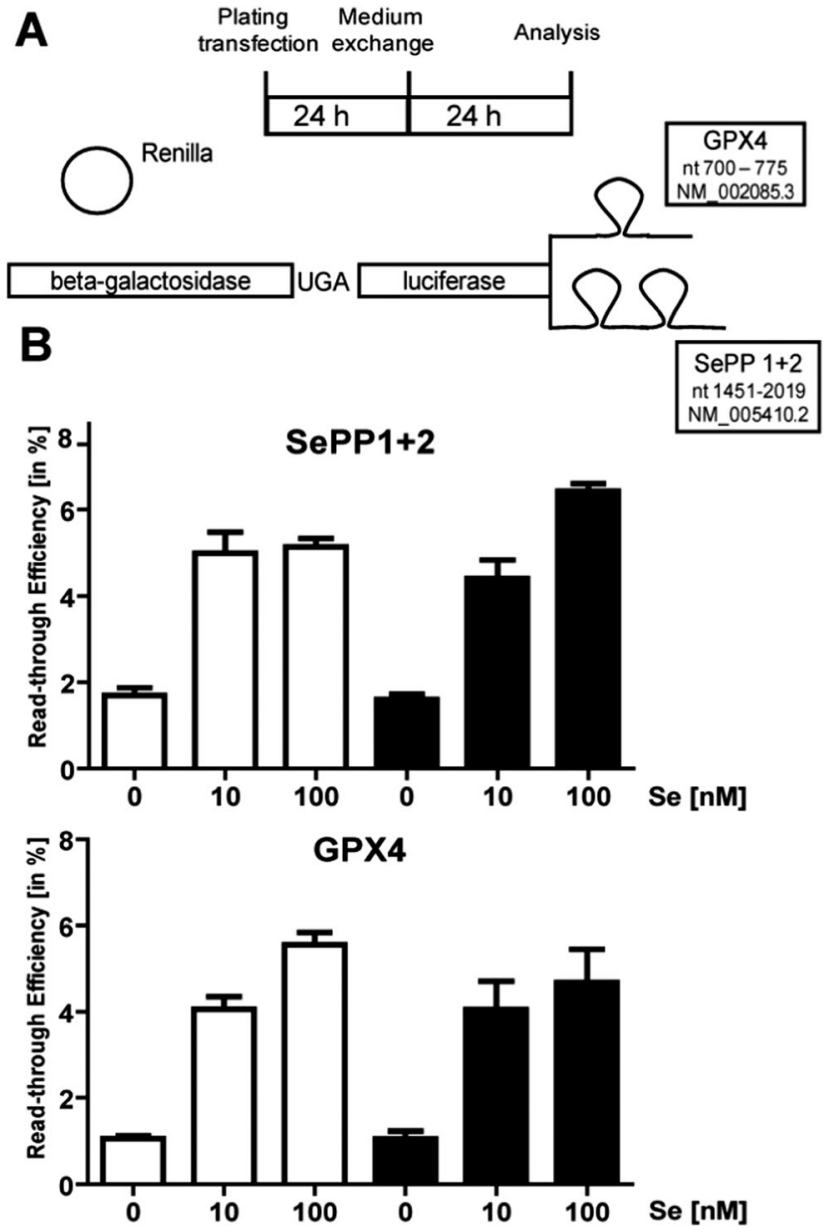

Fig. 3 The effects of hypoxia are not exerted by affecting SECISdependent $\mathrm{Sec}$ insertion. (A) Experimental setup specifying treatment and time schedule, reporter constructs and SECIS element sequences chosen for analysis. (B) Read-through efficiencies under normoxic (white bars) or hypoxic (black bars) conditions in the presence or absence of supplemental selenite ( $n=8$ per experiment). Oxygen supply had no impact on read-through, but selenite increased the SECIS-dependent Sec insertion rates.

under low oxygen supply. The pBLUGA reporter system was chosen to assess the effects of hypoxia on the co-translational insertion of Sec into growing proteins (Fig. 3A). Biosynthesis of the gal-luc fusion protein was increased by selenite supplementation using the SECIS elements of GPX4 or SePP (Fig. 3B). However, hypoxia was without effect on Sec insertion using this reporter system. Next, signalling via the HIF pathway was tested as a likely route mediating the effects of hypoxia on selenoprotein biosynthesis. Constitutive active HIF1 $\alpha$ or HIF $2 \alpha$ variants were co-transfected and strongly induced the activity of a HIF-reporter construct (HRE-Luc) irrespective of the actual Se concentration (Fig. 4A). Again, SePP levels in the medium (Fig. 4B), or GPX4 activity in cell homogenates remained unaffected by constitutively activated HIF signalling (Fig. 4C). Similarly, when a dominant negative HIF1 $\beta$ variant $(\triangle \mathrm{ARNT})$ was co-transfected, the activity of the HIF-reporter declined (Fig. 4D), but SePP expression was not affected (Fig. 4E and F). These findings 
A

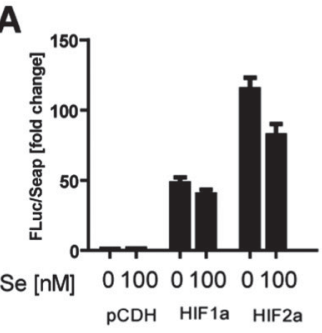

C

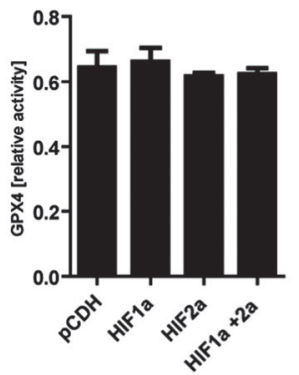

E
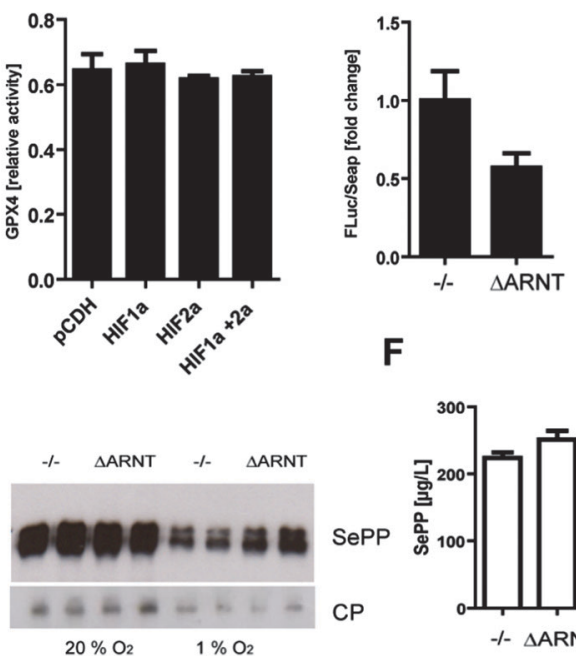

$\mathbf{F}$

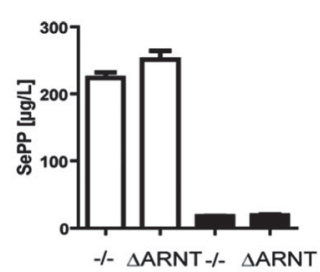

Fig. 4 The effects of hypoxia are independent of HIF signalling. (A) The active variants of HIF1 and HIF2 induce the hypoxia-responsive positive control reporter gene independent of the Se status. (B, C) SePP protein levels in culture medium and GPX4 activity in cell homogenates are not affected by stimulating the HIF pathway with the constitutively active HIF variants. (D) $\triangle A R N T$ is a negative regulator of the hypoxia pathway. Transfection of $\triangle A R N T$ into HepG2 cells reduces activity of a hypoxia reporter gene. (E, F) Inhibition of HIF signalling by $\triangle$ ARNT does not affect immunoreactive SePP levels in medium as tested by western blot or immunoluminometric assay. Representative data from $n=2-4$ similar experiments each involving at least duplicate determinations are shown.

indicate that neither altered SECIS-dependent Sec insertion nor HIF-signalling is responsible for the effects of hypoxia on the expression of SePP and GPX4.

\section{Regulation of selenoprotein biosynthesis factors by hypoxia}

Besides the cis-acting SECIS elements, a set of Sec-specific enzymes and trans-acting factors controls the extent of selenoprotein biosynthesis which may become limiting under pathological conditions. Therefore, effects of hypoxia with or without supplemental Se were determined on the expression level of seven selenoprotein biosynthesis-related genes. Two hypoxiaresponsive controls, i.e., carbonic anhydrase 9 (CA9) and phosphoglycerate kinase (PGK), verified the experimental setup and the successful induction of hypoxia (Fig. 5A and B).

Under these conditions, no significant oxygen-dependent effects were observed for SBP2, SEPSECS or TRIT1 gene expression (Fig. 5C-E). In comparison, transcript concentrations of EFsec increased strongly upon selenite supplementation under normoxic conditions but not in hypoxia (Fig. 5F). Similar intense
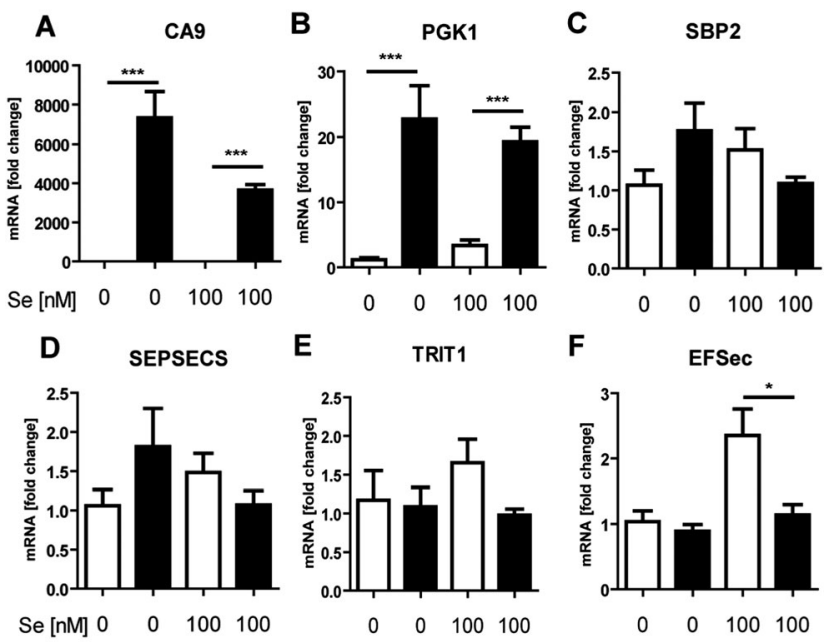

E

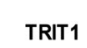

$\mathbf{F}$
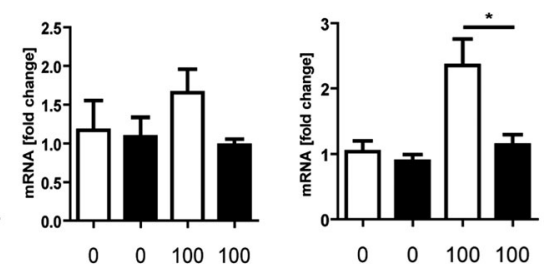

G

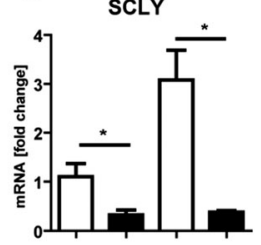

Se $[\mathrm{nM}] \quad 0 \quad 0 \quad 100100$

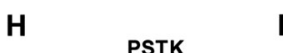

I SEPHS2

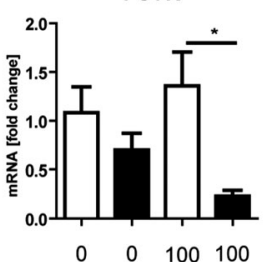

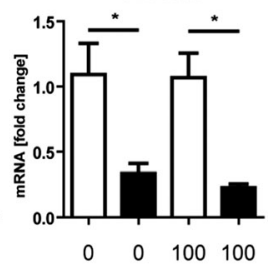

Fig. 5 Hypoxia down-regulates key factors of the selenoprotein biosynthesis machinery. HepG2 cells were cultivated under normoxic and hypoxic conditions, and mRNA concentrations were determined by qRTPCR ( $n=4$ per experiment). Characteristic induction of the two positive HIF-regulated control genes (A) CA9 and (B) PGK1 is observed. Transcripts encoding central factors and proven bottlenecks of selenoprotein biosynthesis are reduced by hypoxia in a gene-specific way; (F) EFSec, (G) SCLY, (H) PSTK, and (I) SEPHS2. The other central selenoprotein biosynthesis factors tested (C, SBP2; D, SEPSECS; E, TRIT1) are similarly expressed under normoxic and hypoxic conditions.

effects of hypoxia were elicited on the mRNA concentrations of SCLY, PSTK, and SEPHS2 which all were strongly down-regulated as compared to normoxic conditions (Fig. 5G-I). PSTK is an established rate-limiting factor for selenoprotein biosynthesis. ${ }^{20}$ As SEPHS2 is a selenoprotein itself and its mRNA is down-regulated by hypoxia, it likely becomes severely affected under hypoxic conditions. A reduced expression of SCLY reduces the rate of Se liberation from degraded selenoproteins thereby negatively affecting substrate availability for selenoprotein biosynthesis. ${ }^{21}$ Collectively, these findings are compatible with the notion that selenoprotein biosynthesis is strongly impaired under hypoxic conditions by a concerted down regulation of crucial enzymes needed for efficient Se recycling, Se activation and generation of Sec-loaded tRNA. Preserving the mRNA concentrations of SBP2, EFsec and SEPSECS implies that the adaptation process to hypoxia is not intended to shut down the biosynthesis of selenoproteins completely.

\section{Discussion}

Hypoxia is a severe threat to living cells inducing a number of profound metabolic adaptations aiming at preserving cell 
integrity, energy supply and functionality. Our results indicate a strong modulating effect of hypoxia on the expression pattern of selenoproteins in favour of GPX4 and at the expense of DIO1, SePP and other GPX isozymes. Hypoxia thus shifts the cellular metabolism from energy-consuming dispensable altruistic pathways towards an emergency program necessary for coping with the more challenging hypoxic metabolism.

Why may these effects constitute a reasonable and sensible response for a cell under low oxygen supply?

Among the different GPX isozymes, GPX4 is the only essential enzyme ubiquitously expressed and essentially needed to protect cells from oxidative stress-induced cell death. ${ }^{22}$ In comparison, GPX1, GPX2, and GPX3 are less vital enzymes, implicated in intra- or extracellular peroxide degradation and gastrointestinal functions, respectively. Their activities are not essential, and mice with genetically impaired expression of any of these three GPX isoforms are viable and display mild phenotypes only unless challenged by additional noxae. ${ }^{23}$ The same applies to DIO1 and SePP, which do participate in thyroid hormone metabolism, iodine and Se homeostasis and Se transport, respectively, without being absolutely essential. The general response pattern observed here under hypoxia correlates nicely to the well-established hierarchy of selenoprotein expression under limited Se supply. ${ }^{24}$ Depending on Se availability, biosynthesis of essential selenoproteins is preferred over less-vital selenoproteins in order to sustain essential Se-dependent pathways. $^{2}$ Hypoxia thus constitutes another important general modifier of selenoprotein expression preferences similarly potent as the Se status itself.

Besides essentiality and the shift in selenoprotein expression pattern, the comparison of selenoprotein mRNA and protein concentrations reveals consistently diminished protein concentrations with respect to the available transcript abundance. Even in the case of the up regulated GPX4, a 5-10-times increased transcript concentration under Se depleted conditions becomes translated into a modest 2-3-times higher GPX4 enzymatic activity and immunogenic protein levels, respectively. On the one hand, this finding supports the notion that selenoprotein biosynthesis is generally impaired under low oxygen supply, potentially in order to save energy and shut down unnecessary anabolic pathways; on the other hand, the effects observed indicate that GPX4 mRNA expression is extremely sensitive to oxygen levels overcoming a diminished biosynthesis rate under hypoxic conditions by excessive transcript abundance competing out other selenoprotein mRNAs. Whether this isozyme-specific response to hypoxia is mainly exerted by an increased transcription rate of GPX4 or by posttranscriptional mechanisms stabilizing GPX4 transcripts remains to be elucidated. The data support the general notion that selenoproteins differ profoundly from regular proteins as the posttranscriptional regulatory mechanisms are powerful and necessitate the quantification of both mRNA levels and protein abundance or enzymatic activity levels in order to fully understand their regulation of expression.

It is interesting to note that not all components of the biosynthesis machinery are likewise affected by hypoxia as the transcript abundance of some important factors (SBP2, SEPSECS, and EFsec) is maintained. EFsec and SBP2 are likely retained as their activities are essentially needed for some on-going translation of essential selenoproteins like GPX4.

In comparison, the down regulation of the other components needed for Sec-tRNA generation (PSTK, SEPHS2, SCLY) might not have an immediate impact on selenoprotein mRNA translation as some substrates and Sec loaded tRNA remain available at the beginning of hypoxia. However, chronic hypoxia will likely deplete the cell from the substrates needed for selenoprotein biosynthesis. Then, a limiting expression of these enzymes involved in Sec-tRNA biosynthesis may become critical, compromising even GPX4 biosynthesis and causing severe harm. Another unexpected finding in this respect is the lack of dependence on the mRNA-specific SECIS-elements as they are believed to decisively control the hierarchical expression of selenoproteins independent of the Se availability. ${ }^{25}$ It may be speculated that two powerful alternative routes for a better fine tuning of the preferential biosynthesis of some selenoproteins over others have evolved, i.e., one in response to limited Se and the other in response to low oxygen availability.

The same reason might underlie the obvious independence of the major hypoxia-responsive pathway, i.e., HIF-controlled transcription. Our results with genetically induced or impaired HIF activity support the notion that the classical HIF pathway does not control selenoprotein expression. Our findings nicely correlate with the diminished expression of the Se-dependent thioredoxin reductase mRNA and activity under hypoxic conditions, which is also proved to be independent of HIF activity. ${ }^{26}$ Again, avoiding direct HIF-dependence might allow for a better control and finer adaptation to the actual degree of hypoxia. But of course, these hypotheses need to be experimentally tested in the future.

So, what are likely physiological consequences of the hypoxia-dependent changes in selenoprotein expression?

As discussed above, the cell will profit from reducing anabolic pathways as it saves energy, and from the altered selenoprotein expression program as the essentially needed GPX4 is induced preventing the fast activation of cell-death pathways (Fig. 6). ${ }^{22}$ Systemic consequences are likely governed by the impaired DIO1 and SePP biosynthesis.

DIO1 deficiency may contribute to the deranged thyroid hormone patterns in critical illness, the so-called low T3 syndrome. ${ }^{27}$ These adaptations causing low concentrations of the active thyroid hormone $\mathrm{T} 3$ reduce the basal metabolic rate and thus oxygen consumption, thereby alleviating the limited oxygen supply under hypoxia. Reduced SePP biosynthesis is known to cause a decline in Se concentrations in serum, best characterized during an acute-phase response or during sepsis., ${ }^{9,15,20,28}$ This response may be needed to support the increased intracellular demand for biosynthesis of essential selenoproteins as explained above, and at the same time deprive the circulation form SePP which might otherwise interfere with ROS-mediated defence strategies against invading bacteria in sepsis, and shutting down a dispensable pathway in an emergency setting, i.e., Se transport, which is obviously not of immediate urgency under hypoxia. 

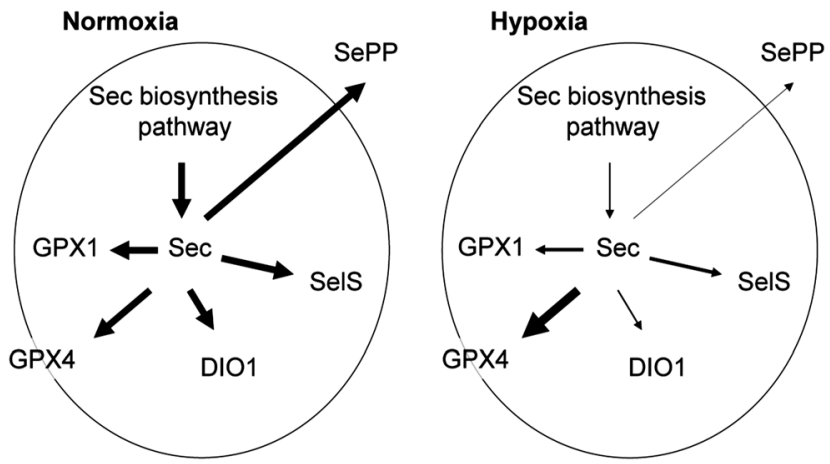

Fig. 6 Schematic overview of effects of hypoxia on the selenoprotein expression pattern in hepatocytes. In normoxia, dietary Se is taken up and used to support $\mathrm{Sec}$ and consequently biosynthesis of all selenoproteins. Secreted SePP constitutes a major fraction and supplies other tissues with the essentially needed trace element. Under hypoxia, hepatocytes reduce the anabolic pathways generating Sec-loaded tRNA and thus net selenoprotein biosynthesis. GPX4 constitutes an exception, as GPX4 mRNA accumulates strongly thereby out-competing other selenoprotein transcripts for Sec leading to increased GPX4 expression levels and strongly reduced SePP-biosynthesis and release into the circulation.

However, in the long run and especially in view of the powerful intensive care medication at hand, this response of decreased thyroid hormone availability and low serum se concentrations resulting in target tissue Se deficiency will constitute a severe complication for fast convalescence and survival. Hence, this interaction may explain the increased survival rates of Se-replete or actively supplemented sepsis ${ }^{9,28}$ and cancer $^{29}$ patients, and the improved convalescence of cardiac arrest patients upon Se supplementation. ${ }^{11}$ Again, these hypotheses need to be rigorously tested in future clinical trials.

\section{Conclusions}

Our results indicate that Se export via SePP becomes shut down by hypoxia in favour of increased intracellular GPX4 expression thereby shifting the balance from regular Se metabolism needed for ubiquitous biosynthesis of all selenoproteins towards an emergency program preferring essential selenoproteins. This shifted pattern of selenoprotein biosynthesis in hepatocytes may underlie the declining Se status and low SePP levels as observed in some human pathologies including severe illness, ${ }^{30}$ sepsis, ${ }^{15}$ trauma, cirrhosis, ${ }^{31}$ hepatitis, ${ }^{31}$ dialysis,${ }^{32}$ cancer, ${ }^{33}$ or infections. ${ }^{34}$ Supplemental Se increased selenoprotein expressions to a certain extent in our experiments. This finding supports the concept of an adjuvant treatment via Se supplementation already tested in the clinics, e.g. in severe sepsis $^{9}$ or after cardiac arrest. ${ }^{11}$

\section{Acknowledgements}

We thank Katja Schreiber, Carola Geiler and Vartiter Seher for excellent technical assistance. The study was supported by the Deutsche Forschungsgemeinschaft (DFG; GraKo 1208, SCHO849/4-1, RE3038/1-1) and Federal Ministry of Economics and Technology (BMWi; KF2263202CS2). Finally, the authors would like to express their gratitude to an anonymous reviewer for his/her constructive remarks on their study.

\section{References}

1 A. V. Lobanov, D. L. Hatfield and V. N. Gladyshev, Biochim. Biophys. Acta, 2009, 1790, 1424-1428.

2 L. Schomburg and U. Schweizer, Biochim. Biophys. Acta, 2009, 1790, 1453-1462.

3 M. P. Rayman, Lancet, 2012, 379, 1256-1268.

4 C. D. Davis, P. A. Tsuji and J. A. Milner, Annu. Rev. Nutr., 2012, 32, 73-95.

5 O. A. Levander and M. A. Beck, Br. Med. Bull., 1999, 55, 528-533.

6 C. Berr, J. Arnaud and T. N. Akbaraly, BioFactors, 2012, 38, 139-144.

7 H. Koyama, R. Abdulah, T. Ohkubo, Y. Imai, H. Satoh and K. Nagai, Nutr. Res., 2009, 29, 94-99.

8 L. Schomburg, Nat. Rev. Endocrinol., 2012, 8, 160-171.

9 M. W. Angstwurm, L. Engelmann, T. Zimmermann, C. Lehmann, C. H. Spes, P. Abel, R. Strauss, A. MeierHellmann, R. Insel, J. Radke, J. Schüttler and R. Gärtner, Crit. Care Med., 2007, 35, 118-126.

10 S. L. Mehta, S. Kumari, N. Mendelev and P. A. Li, BMC Neurosci., 2012, 13, 79.

11 J. Reisinger, K. Hollinger, W. Lang, C. Steiner, T. Winter, A. Winter, M. Mori, A. Lindorfer, D. Kiblbock and P. Siostrzonek, Am. J. Emerg. Med., 2009, 27, 176-181.

12 L. Schomburg, U. Schweizer, B. Holtmann, L. Flohé, M. Sendtner and J. Köhrle, Biochem. J., 2003, 370, 397-402.

13 R. A. Lawrence and R. F. Burk, Biochem. Biophys. Res. Commun., 1976, 71, 952-958.

14 K. Renko, C. S. Hoefig, F. Hiller, L. Schomburg and J. Köhrle, Endocrinology, 2012, 153, 2506-2513.

15 B. Hollenbach, N. G. Morgenthaler, J. Struck, C. Alonso, A. Bergmann, J. Köhrle and L. Schomburg, J. Trace Elem. Med. Biol., 2008, 22, 24-32.

16 P. J. Hofmann, L. Schomburg and J. Köhrle, Toxicol. Sci., 2009, 110, 125-137.

17 H. Kollmus, L. Flohe and J. E. McCarthy, Nucleic Acids Res., 1996, 24, 1195-1201.

18 D. E. Handy, G. Hang, J. Scolaro, N. Metes, N. Razaq, Y. Yang and J. Loscalzo, J. Biol. Chem., 2006, 281, 3382-3388.

19 T. L. Clanton, J. Appl. Phys., 2007, 102, 2379-2388.

20 K. Renko, P. J. Hofmann, M. Stoedter, B. Hollenbach, T. Behrends, J. Köhrle, U. Schweizer and L. Schomburg, FASEB J., 2009, 23, 1758-1765.

21 S. Kurokawa, M. Takehashi, H. Tanaka, H. Mihara, T. Kurihara, S. Tanaka, K. Hill, R. Burk and N. Esaki, J. Nutr. Sci. Vitaminol., 2011, 57, 298-305.

22 A. Seiler, M. Schneider, H. Forster, S. Roth, E. K. Wirth, C. Culmsee, N. Plesnila, E. Kremmer, O. Radmark, W. Wurst, G. W. Bornkamm, U. Schweizer and M. Conrad, Cell Metab., 2008, 8, 237-248. 
23 M. V. Kasaikina, D. L. Hatfield and V. N. Gladyshev, Biochim. Biophys. Acta, 2012, 1823, 1633-1642.

24 G. Bermano, F. Nicol, J. A. Dyer, R. A. Sunde, G. J. Beckett, J. R. Arthur and J. E. Hesketh, Biochem. J., 1995, 311(Pt 2), 425-430.

25 S. C. Low, E. Grundner-Culemann, J. W. Harney and M. J. Berry, EMBO J., 2000, 19, 6882-6890.

26 S. Naranjo-Suarez, B. A. Carlson, P. A. Tsuji, M. H. Yoo, V. N. Gladyshev and D. L. Hatfield, PLoS One, 2012, 7, e30470.

27 R. Gärtner, J. Trace Elem. Med. Biol., 2009, 23, 71-74.

28 X. Forceville, J. Trace Elem. Med. Biol., 2007, 21(suppl 1), 62-65.
29 H. A. Meyer, T. Endermann, C. Stephan, M. Stoedter, T. Behrends, I. Wolff, K. Jung and L. Schomburg, PLoS One, 2012, 7, e46644.

30 X. Forceville, D. Vitoux, R. Gauzit, A. Combes, P. Lahilaire and P. Chappuis, Crit. Care Med., 1998, 26, 1536-1544.

31 M. Navarro-Alarcon, H. Lopez-Ga de la Serrana, V. PerezValero and M. C. Lopez-Martinez, Sci. Total Environ., 2002, 291, 135-141.

32 M. Tonelli, N. Wiebe, B. Hemmelgarn, S. Klarenbach, C. Field, B. Manns, R. Thadhani and J. Gill, BMC Med., 2009, 7, 25.

33 N. Rohwer, C. Zasada, S. Kempa and T. Cramer, Oncogene, 2013, 32, 3569-3576.

34 M. A. Beck, O. A. Levander and J. Handy, J. Nutr., 2003, 133, 1463S-1467S. 